\title{
Monocyte upregulation of podoplanin during early sepsis induces complement inhibitor release to protect liver function
}

\author{
Zhanli Xie, ${ }^{1}$ Bojing Shao, ${ }^{2}$ Christopher Hoover, ${ }^{2,3}$ Michael McDaniel, ${ }^{2}$ Jianhua Song, ${ }^{2}$ Miao Jiang, \\ Zhenni Ma, ${ }^{1}$ Fei Yang, ${ }^{1}$ Jingjing Han, ${ }^{1}$ Xia Bai, ${ }^{1,4,5}$ Changgeng Ruan, ${ }^{1,4,5}$ and Lijun Xia ${ }^{1,2,3,4}$ \\ Jjiangsu Institute of Hematology, National Clinical Research Center for Hematologic Diseases, NHC Key Laboratory \\ of Thrombosis and Hemostasis, The First Affiliated Hospital of Soochow University, Jiangsu, China. ${ }^{2}$ Cardiovascular \\ Biology Research Program, Oklahoma Medical Research Foundation, Oklahoma City, Oklahoma, USA. ${ }^{3}$ Department of \\ Biochemistry and Molecular Biology, University of Oklahoma Health Sciences Center, Oklahoma City, Oklahoma, USA. \\ ${ }^{4}$ Collaborative Innovation Center of Hematology and ${ }^{5}$ State Key Laboratory of Radiation Medicine and Protection, Soochow \\ University, Suzhou, China.
}

Multiple organ failure in sepsis is a progressive failure of several interdependent organ systems. Liver dysfunction occurs early during sepsis and is directly associated with patient death; however, the underlying mechanism of liver dysfunction is unclear. Platelet transfusion benefits patients with sepsis, and inhibition of complement activation protects liver function in septic animals. Herein, we explored the potential link between platelets, complement activation, and liver dysfunction in sepsis. We found that deletion of platelet C-type lectin-like receptor 2 (CLEC-2) exacerbated liver dysfunction in early sepsis. Platelet CLEC-2-deficient mice exhibited higher complement activation, more severe complement attack in the liver, and lower plasma levels of complement inhibitors at early time points after $E$. coli infection. Circulating monocytes expressed the CLEC-2 ligand podoplanin in early sepsis, and podoplanin binding induced release of complement inhibitors from platelets. Injection of complement inhibitors released from platelets reduced complement attack and attenuated liver dysfunction in septic mice. These findings indicate a new function of platelets in the regulation of complement activation during sepsis.

Authorship note: $Z X$ and BS contributed equally to this study.

Conflict of interest: The authors have declared that no conflict of interest exists.

Copyright: (c) 2020, American Society for Clinical Investigation.

Submitted: November 4, 2019

Accepted: June 3, 2020

Published: July 9, 2020.

Reference information: JCl Insight. 2020;5(13):e134749.

https://doi.org/10.1172/jci.

insight.134749.

\section{Introduction}

Sepsis is a life-threatening organ dysfunction triggered by a dysregulated host response to infection (1). The incidence of sepsis is over 1.5 million cases annually in the United States (2), and approximately 18 million cases globally (3). Around $30 \%$ of patients with sepsis die of multiple organ dysfunction or failure in intensive care units (4). In sepsis, the liver is injured by inflammatory cytokines, microthrombi, and pathogens, leading to liver dysfunction and failure. The injured liver, in turn, facilitates dysfunction of other organs by enhancing inflammatory responses (5) and activating coagulation (6), whereby liver injury directly contributes to the death of patients with sepsis (7). Liver dysfunction occurs at an early stage of sepsis (8) and is associated with a poor prognosis (9); therefore, protecting liver function in early sepsis may prevent liver injury from progressing to liver failure, thus increasing patient survival. However, the mechanism underlying liver dysfunction in sepsis is poorly understood. Thus, few interventions to treat septic liver injury are available.

Platelets, the critical player in hemostasis and thrombosis, have essential functions in innate immunity (10) and tissue homeostasis (11). In sepsis, activated platelets secrete many proinflammatory and prothrombotic mediators that enhance organ injury (12). However, inhibition of platelet activation exhibits inconsistent therapeutic benefits in sepsis $(13,14)$. By contrast, platelet transfusion into nonbleeding sepsis patients shows a clinical benefit $(15,16)$ and reduces the mortality of $E$. coli-injected mice $(17)$. The protective effect of platelet transfusion depends on platelet receptor C-type lectin-like receptor 2 (CLEC-2) (18), although its underlying mechanisms remain elusive.

CLEC-2 is a membrane receptor mainly expressed on platelets and primarily functions in the separation of lymphatic vessels from veins during development, maintenance of blood vessel integrity in some organs during inflammation, and promotion of tumor metastasis (19). Podoplanin, the only known endogenous 
ligand for CLEC-2, is expressed on many cell types, including lymphatic endothelial cells, kidney podocytes, and lung alveolar epithelial cells (19). Blood cells and blood vessel endothelial cells in healthy animals do not express podoplanin (19), whereas resident macrophages in septic animals can express podoplanin (18). It is thought that CLEC-2 binding to podoplanin inhibits inflammatory cytokine generation in resident macrophages, by which septic animals are protected $(18,20)$. Inhibition of inflammation, however, has given disappointing results in clinical trials on sepsis management (21). Thus, the mechanism by which platelets and platelet CLEC-2 protect patients or animals with sepsis is unclear.

As a critical component of innate immunity, the complement system promotes cell lysis and leukocyte recruitment and phagocytosis, in addition to regulating coagulation and cell signaling. There are 3 pathways to activate the complement system: the classical, alternative, and lectin pathways, which converge at the central complement component C3. C3 is enzymatically processed into C $3 \mathrm{a}$ and C3b, and the latter then cleaves C5 into C5a and C5b. Finally, C5b binds C6, C7, C8, and C9 to form the membrane attack complex (C5b-9). In sepsis, the complement system is activated by pathogens (22), and controlled complement activation benefits pathogen clearance (23). However, overactivation of the complement system causes organ damage (24). For example, uncontrolled complement activation injures heart function in a mouse sepsis model (25), whereas inhibition of C3 or C5 protects liver function in a primate model of $E$. coli sepsis (26). Thus, inhibition of complement activation may protect liver function in sepsis. However, the mechanism by which complement activation is autonomously controlled in sepsis is not well known.

Here, we report that platelet CLEC-2 protects liver function in a mouse model of E. coli-induced sepsis. In early sepsis, circulating monocytes expressed podoplanin, which bound to CLEC-2 and induced release of complement inhibitors from platelets. The released complement inhibitors protected the liver from complement attack in early sepsis and reduced subsequent inflammation in the liver. Our findings suggest a potentially novel mechanism by which platelets protect liver function during early sepsis and an important function of platelets in inhibiting complement activation.

\section{Results}

Platelet CLEC-2 deficiency enhances liver dysfunction in early sepsis. We generated platelet-specific Clec-2deficient mice (Clec-2 $2^{f / f l} \mathrm{Pf4}-\mathrm{Cre}$ ) by breeding Clec-2 $2^{f / f l}$ mice with PF4-Cre transgenic mice, which express Cre recombinase specifically in megakaryocytes and platelets (27). To explore whether platelet CLEC-2 protects liver function at the early stage of sepsis, we used Plt Clec2 $2^{-1-}$ mice and the WT littermates. Deletion of platelet CLEC-2 reduced the blood platelet count in male mice, but not female mice, and had no effects on body weight (Supplemental Figure 1, A-C; supplemental material available online with this article; https://doi.org/10.1172/jci.insight.134749DS1). Thus, only female mice were used in in vivo studies. Sepsis was generated in mice by peritoneal injection of a half-lethal dose of E. coli $(2 \times$ $10^{7} \mathrm{CFU} /$ mouse), which presented a slow progression of sepsis, allowing us to detect the alteration of liver function. Challenge with $E$. coli induced similar thrombocytopenia and loss of body weight in WT and Plt Clec2 $2^{-1-}$ mice (Supplemental Figure 2, A and B). The half-lethal dose of E. coli caused the death of around $20 \%$ of WT mice and $70 \%$ of Plt $\mathrm{Clec}^{-1-}$ mice at 100 hours after infection (Figure 1A). Thus, platelet CLEC-2 played a protective role when the mice experienced bacterial infection.

We assessed liver function by detecting the serum levels of alanine transferase (ALT), lactate dehydrogenase (LDH), aspartate aminotransferase (AST), and bilirubin, as well as by examining liver histology. All serum markers of liver function in both WT mice and Plt Clec2 $2^{-1-}$ mice started to increase dramatically at 1 hour after $E$. coli infection (Figure 1, B-E). Liver function markers in WT mice reached a plateau at 4 to 8 hours after infection (Figure 1, B-E). Compared with WT mice, Plt Clec2 ${ }^{-1-}$ mice had significantly higher serum levels of these markers at 8 hours after E. coli injection (Figure 1, B-E). We further examined the liver histology and found that loss of platelet CLEC-2 did not alter the liver histology in healthy mice (Figure 1F). There were considerable histological changes in the liver at 8 hours after $E$. coli infection, including the destruction of hepatic lobules, cytoplasmic vacuolization, sinusoid obstruction, necrosis, and inflammatory cell infiltration (Figure 1F). Here, ballooning degeneration, a form of apoptosis, in the liver was quantified, and platelet CLEC-2 deficiency significantly increased this injury in the liver of infected mice (Figure 1F). These results indicate that deficiency of platelet CLEC-2 exacerbated liver dysfunction in early sepsis.

Platelet CLEC-2 deficiency increases liver inflammation in early sepsis, which is independent of podoplanin-mediated inhibition of cytokine generation in resident macrophages. To study the role of platelet CLEC- 2 in 
A

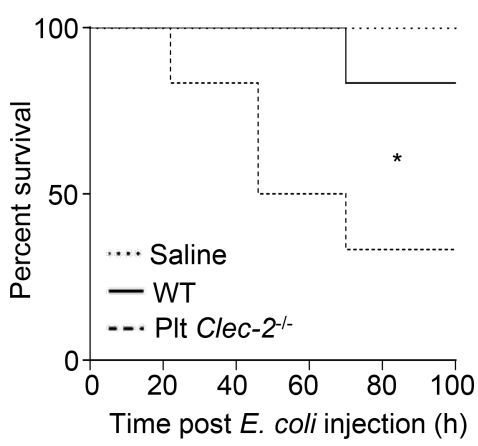

B

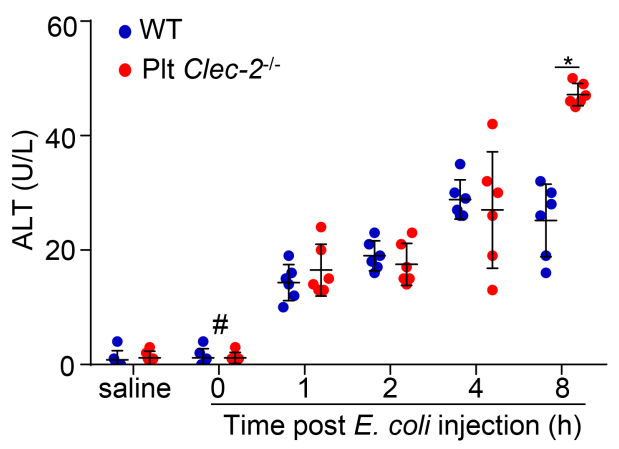

C

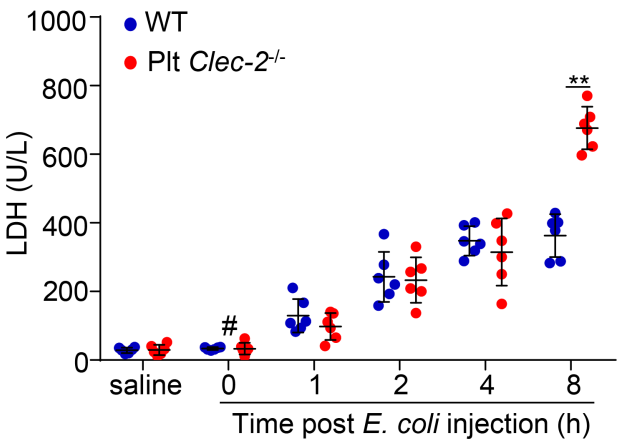

D

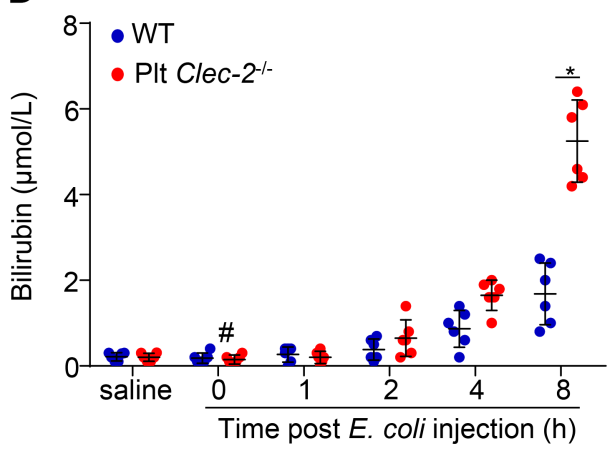

E

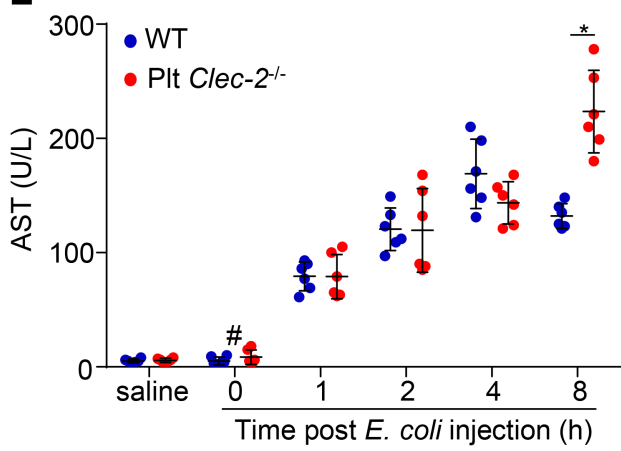

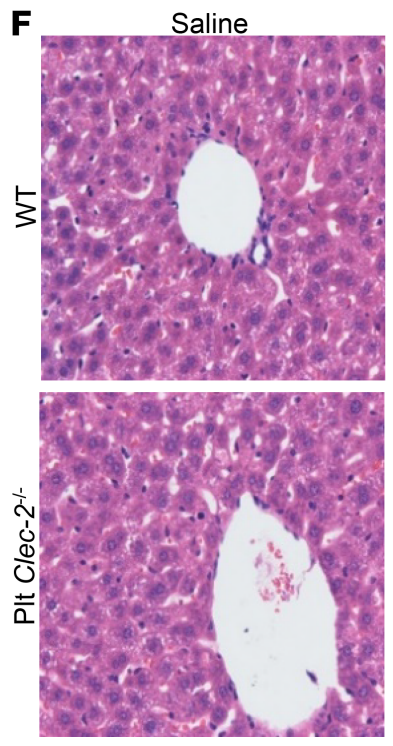
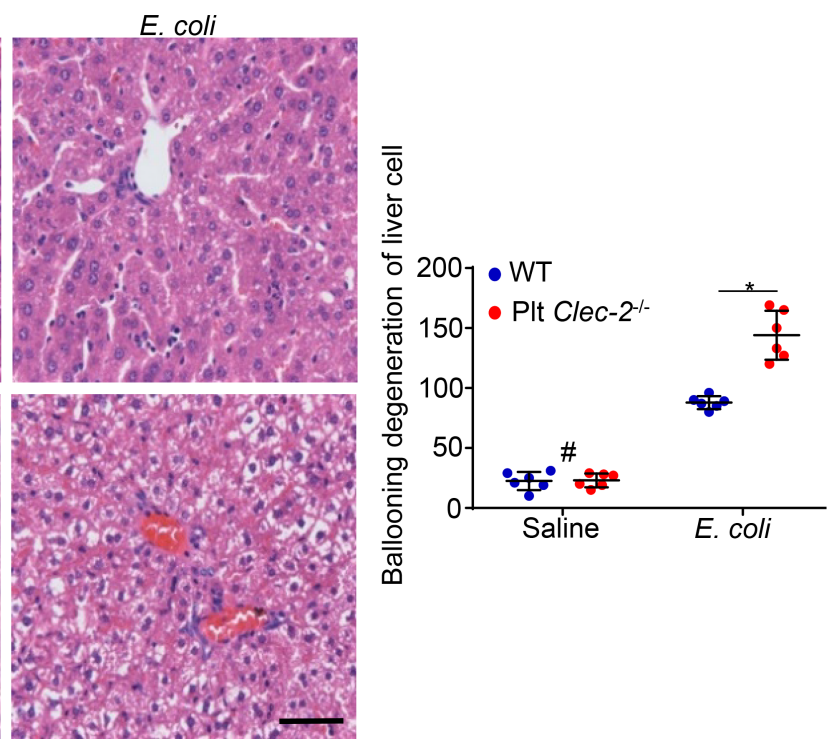

Figure 1. Platelet CLEC-2 deficiency enhances liver dysfunction in early sepsis. (A) Survival rate of mice analyzed using a log-rank test. $n=15$ mice/group. Data were representative of 3 independent experiments. (B-E) Blood chemistry: ALT, LDH, bilirubin, and AST. (F) Representative images and quantification of injuries of H\&E-stained liver sections collected from mice at 8 hours after i.p. injection of saline or $E$. coli. Data are mean \pm SD. $n=6$ mice/group. ${ }^{\#} P<0.05$, comparing the sample at 0 hours of $E$. coli injection with samples at other time points after $E$. coli infection. ${ }^{*} P<0.05$; ${ }^{* *} P<0.01$. Data were analyzed using 1-way ANOVA followed by Tukey's test for multiple groups and were representative of 3 independent experiments. Scale bar: $400 \mu \mathrm{m}$.

inflammation, we examined inflammation responses in the septic liver. WT and Plt $\mathrm{Clec}^{-{ }^{--}}$liver tissues were collected at different time points after E. coli infection, and the expression of TNF- $\alpha$ and IL-1 $\beta$ was analyzed using quantitative PCR. Compared with the liver from healthy mice, the expression of TNF- $\alpha$ and IL-1 $\beta$ in the liver from WT and Plt $\mathrm{Clec}^{-/-}$mice began to increase at 2 hours following $E$. coli infection (Figure 2, A and C). At 8 hours after E. coli infection, the expression of TNF- $\alpha$ and IL-1 $\beta$ in the liver from Plt $\mathrm{Clec}^{---}$mice was significantly higher than that in the liver of WT mice (Figure 2, A and C). TNF- $\alpha$ and IL- $1 \beta$ staining were performed using cryosections of liver tissues collected at 8 hours 
after $E$. coli infection based on published methods (28-30), revealing that CLEC-2 deficiency increased the expression of TNF- $\alpha$ and IL-1 $\beta$ in the liver of infected mice, which partially overlapped with macrophages (F4/80-positive) (Figure 2, B and D; and Supplemental Figure 3, A and B). Thus, platelet CLEC2 deficiency enhanced inflammation in the liver in early sepsis.

Podoplanin is expressed in resident macrophages in the lung of septic mice (20) and cultured macrophages upon lipopolysaccharide stimulation (31), the ligand for CLEC-2 (19). CLEC-2 binding to podoplanin inhibits the generation of inflammatory cytokines in lung macrophages (20). To determine whether podoplanin is expressed in the liver, liver cryosections from E. coli-infected WT mice were stained with antibodies against podoplanin and macrophages. As the positive control, the kidneys, which express podoplanin on podocytes in glomeruli (19), showed strong podoplanin staining (Supplemental Figure 4A). However, there was only weak staining of podoplanin in the WT liver after E. coli injection, which was not colocalized with liver macrophages (Figure 2E and Supplemental Figure 4B). We then examined podoplanin expression on neutrophils, which were the first immune cells to infiltrate the inflamed tissue. An increased number of Ly6G-positive neutrophils was found in the WT liver after E. coli injection, but these infiltrated neutrophils did not express podoplanin (Supplemental Figure 4C). Further confocal imaging revealed more CD41-positive platelets in the liver after E. coli injection; however, these platelets appeared not to interact with podoplanin at the examined time point (Supplemental Figure 5). Thus, our results show that $E$. coli infection did not induce podoplanin expression on the liver macrophages and that platelets did not interact with podoplanin in the liver during early sepsis. These data indicate that increased inflammation in the liver of Plt $\mathrm{Clec}^{-/-}$mice in early sepsis was not due to a lack of podoplanin-dependent inhibition of the macrophage cytokine generation.

Platelet CLEC-2 deficiency increases complement activation and liver attack in early sepsis. Because the complement system plays a key role in the pathogenesis of sepsis (22), we asked whether platelet CLEC-2 is involved in complement activation in early sepsis. First, we validated assays (Supplemental Figure 6) for measuring complement activation products, including C3a, C5a, and C5b-9. Then the activated complement products in plasma from E. coli-injected mice were measured. The plasma levels of C3a, C5a, and C5b-9 were elevated at 1 hour after $E$. coli infection, and reached a plateau at 4 hours after infection in both WT and Plt Clec2 $2^{--}$mice (Figure 3, A-C). Interestingly, the plasma levels of C3a and C5a in Plt Clec2-mice were significantly higher than those in WT mice by 2 hours after $E$. coli infection, whereas the plasma level of C5b-9 in Plt Clec2 ${ }^{-1-}$ mice was higher only at 4 hours after infection (Figure 3, A-C). Thus, loss of platelet CLEC-2 increased complement activation in early sepsis.

We then detected the complement attack in the liver by staining C5b-9 on cryosections. Compared with the liver of saline-injected mice, the liver from mice infected with $E$. coli for 8 hours showed a positive staining for C5b-9, which was stronger in Plt Clec2-- mice (Figure 3D and Supplemental Figure 7). C5b-9 staining was largely colocalized with F4/80-positive macrophages (Figure 3D and Supplemental Figure 7 ), suggesting that complement attacks macrophages as well as other types of liver cells. Meanwhile, $E$. coli infection caused increased cell apoptosis in the liver of Plt Clec2 $2^{-/}$mice compared with WT mice, as demonstrated by TUNEL staining (Figure 3E and Supplemental Figure 8).

CLEC-2 deficiency reduces release of complement inhibitors from platelets in early sepsis. Platelet $\alpha$-granules contain complement inhibitors C1-INH (32), clusterin (33), and factor H (34). To test whether released $\alpha$-granule contents inhibit complement activation, we measured the plasma levels of complement inhibitors in septic mice. The plasma levels of C1-INH, clusterin, and factor $\mathrm{H}$ in WT mice started to increase at 1 hour after $E$. coli infection and reached a plateau around 2-4 hours after infection (Figure 4, A-C). Surprisingly, the plasma levels of these inhibitors in Plt Clec2 $2^{-/}$mice were lower than those in WT mice at multiple time points (Figure 4, A-C). Increased plasma levels of platelet factor 4 (PF4) and P selectin, components of platelet $\alpha$-granules, and P selectin on the platelet surface indicated platelet activation and release. We found that changes in these parameters in WT mice and Plt $\mathrm{Clec}^{-1-}$ mice after $E$. coli infection correlated with the alteration of complement inhibitors at early time points after E. coli injection (Figure 4, D-F). By contrast, the levels of ATP, which is a component of the platelet-dense granule, in platelets of WT and CLEC-2-deficient mice were comparable (Figure 4G). The plasma level of C1-INH, but not that of clusterin and factor $\mathrm{H}$, increased mildly in Plt Clec-2 ${ }^{-/-}$mice 8 hours after E. coli injection (Supplemental Figure 9, A-C), whereas the plasma level of PF4 decreased 8 hours after infection (Supplemental Figure 9D). These results indicate that complement inhibitors were primarily released from platelet $\alpha$-granules upon activation during early sepsis, 
A

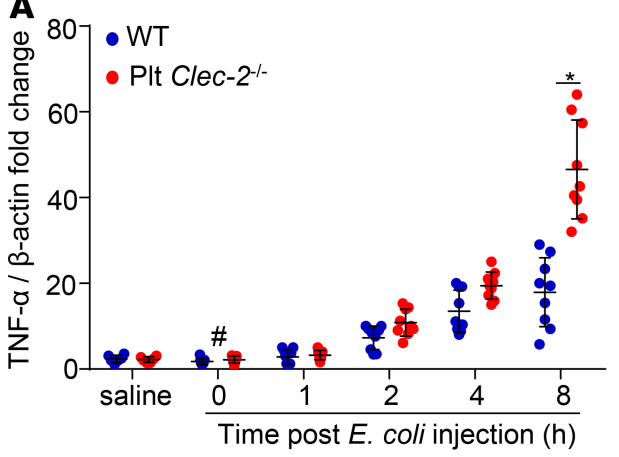

C

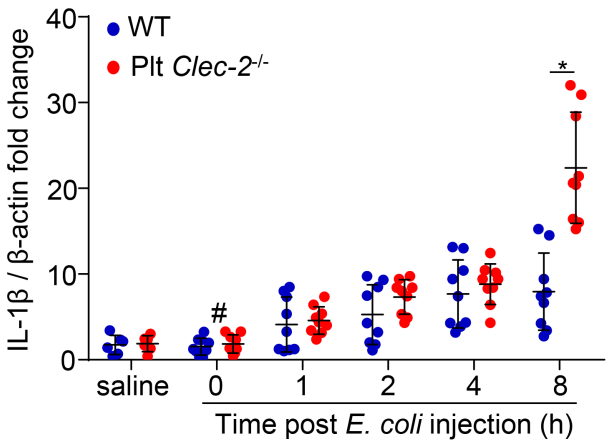

E

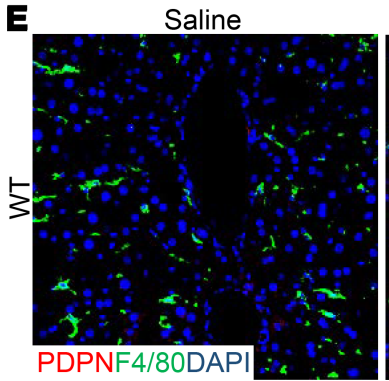

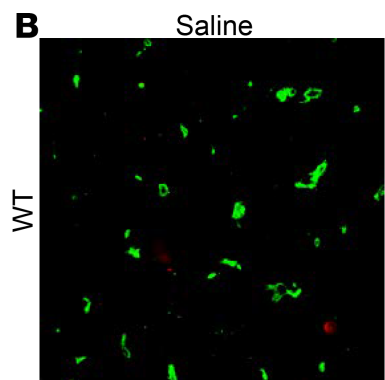

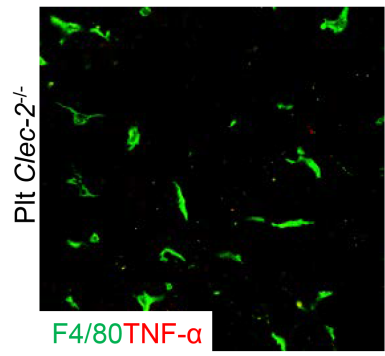

D
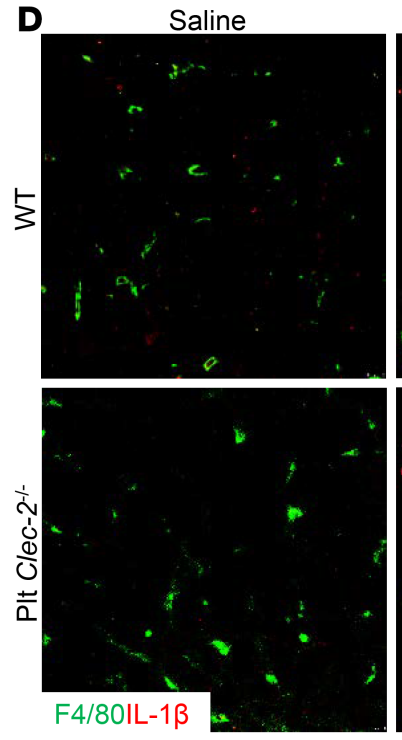

E. coli
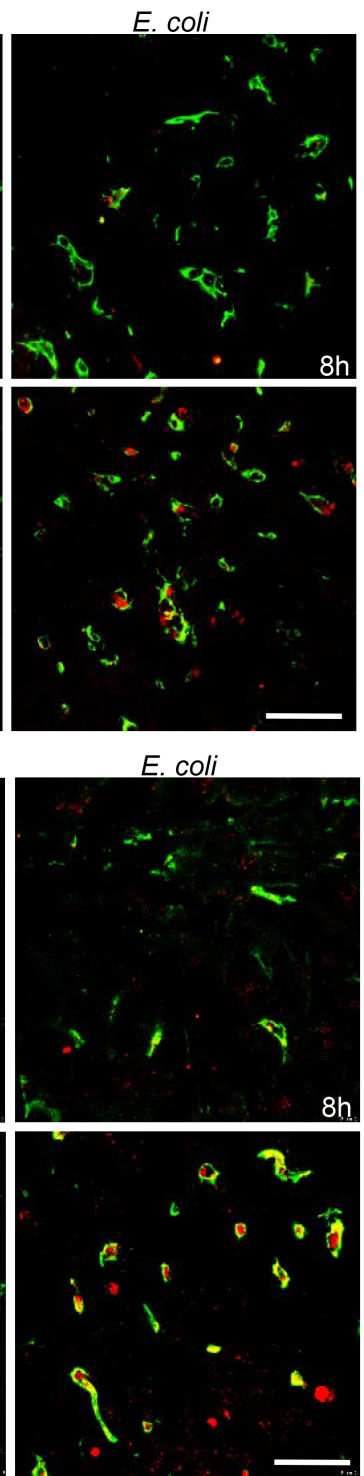

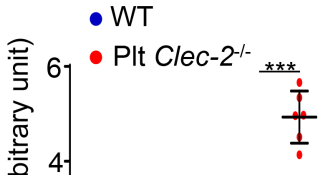

$\frac{p}{1}$
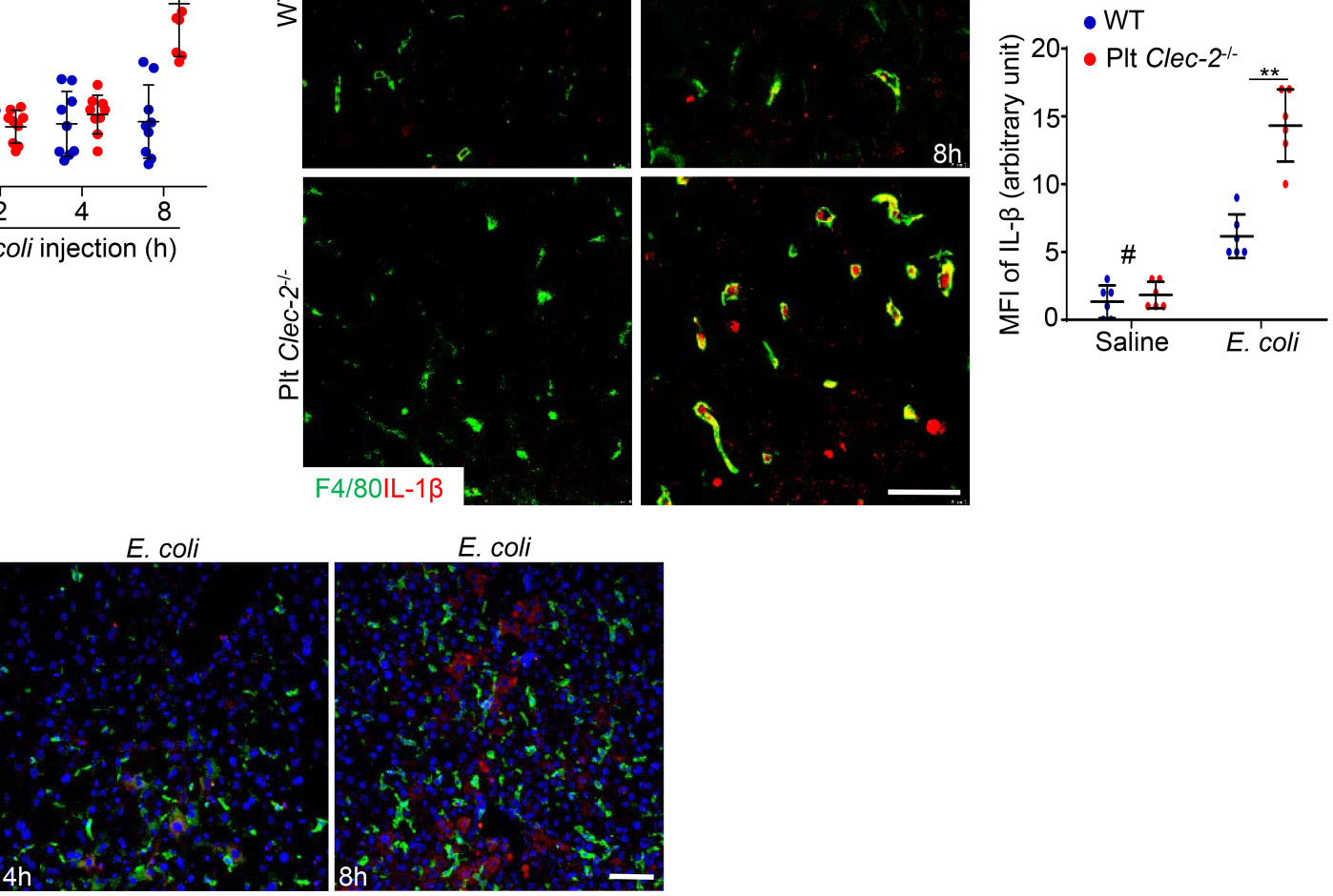

Figure 2. Platelet CLEC-2 deficiency increases liver inflammation in early sepsis. (A and C) mRNA transcripts of Tnfa and IL1B in the livers of WT and PIt Clec $-2^{-/-}$mice. The data represent mean \pm SD. $n=9$ mice/group. (B and D) Representative immunofluorescent images of cryosections of WT and PIt Clec$2^{-1-}$ livers at 8 hours after injection of saline or E. coli. $n=6$ mice/group. Bar graph on the right is quantification of mean fluorescence intensity (MFI) of the anti-TNF- $\alpha$ or -IL-1 $\beta$ staining. Scale bar: $10 \mu \mathrm{m}$. (E) Representative immunofluorescent images of cryosections of WT livers. $n=5$ mice/group. ${ }^{\#} P<0.05$, comparing the sample at 0 hours of $E$. coli injection with samples at other time points after $E$. coli infection. ${ }^{*} P<0.05$; ${ }^{* * *} P<0.001$. Data were analyzed using 1-way ANOVA followed by Tukey's test for multiple groups and were representative of 3 independent experiments. Scale bar: $25 \mu \mathrm{m}$.

and at later time points, cell sources other than platelets may have also contributed to the release of some inhibitors such as C1-INH. Interaction of podoplanin and CLEC-2 is known to induce release of platelet $\alpha$-granules; thus, our data indicate that CLEC-2 deficiency impaired the release of platelet $\alpha$-granules in early sepsis. 
A

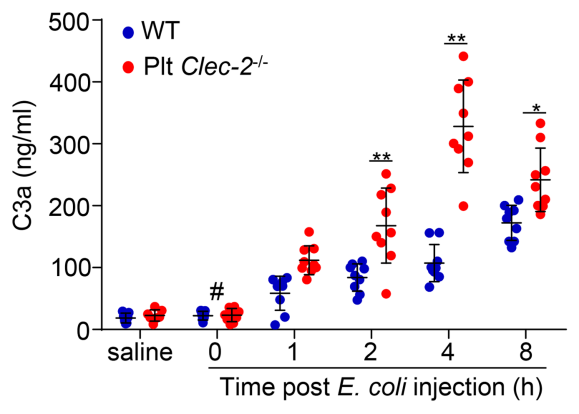

B

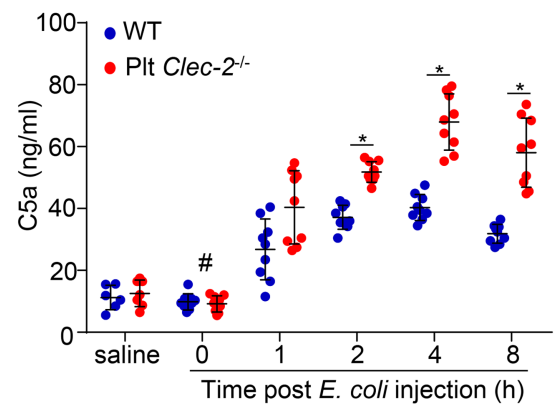

C

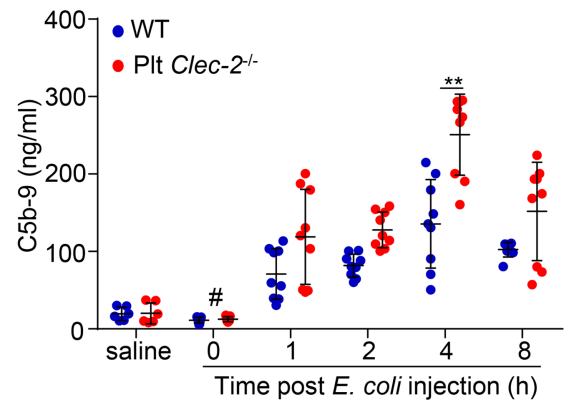

D
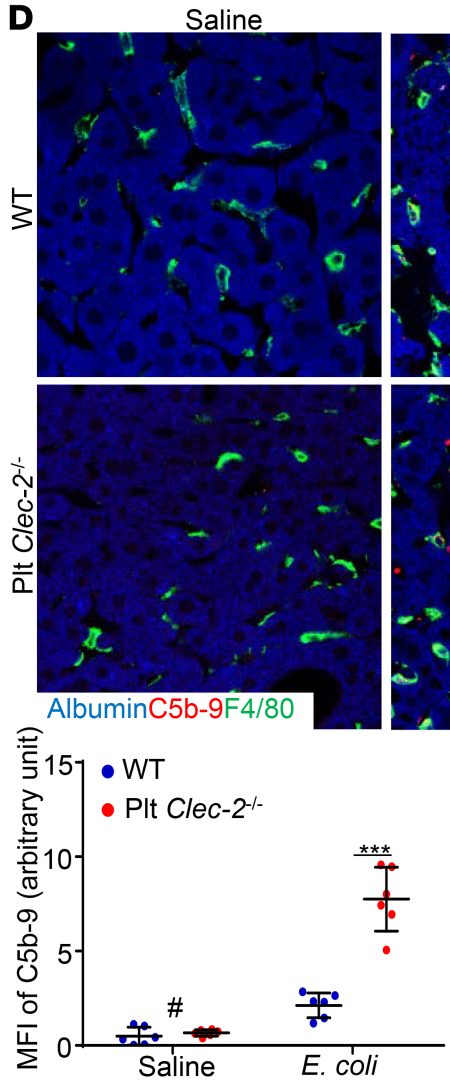

E. coli

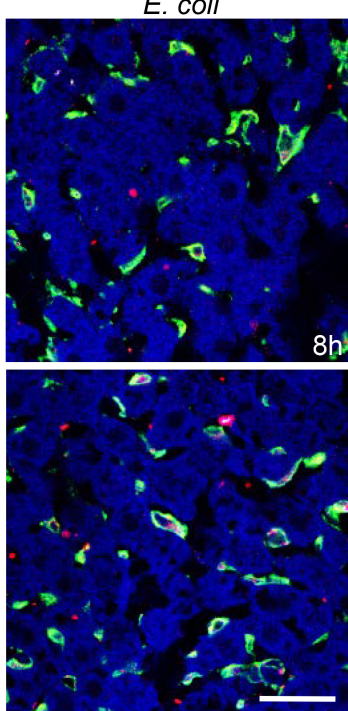

E
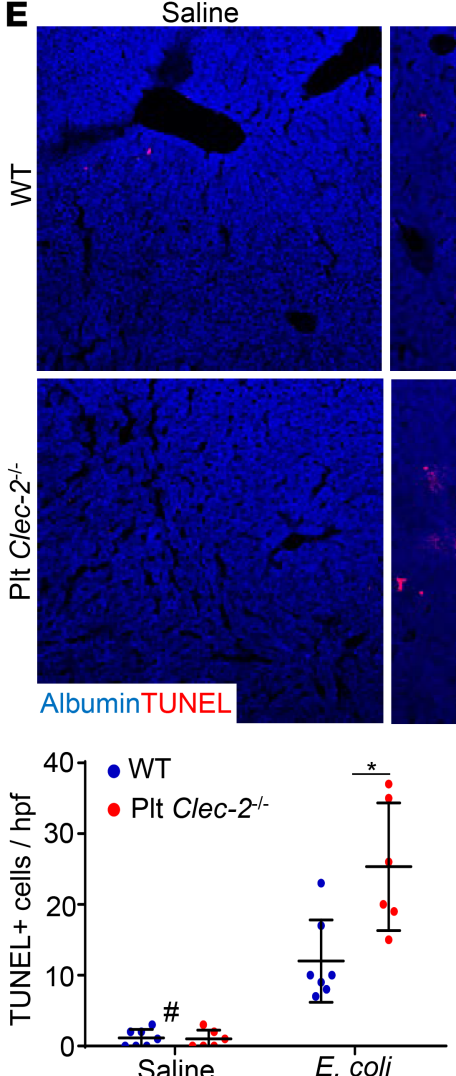

E. coli

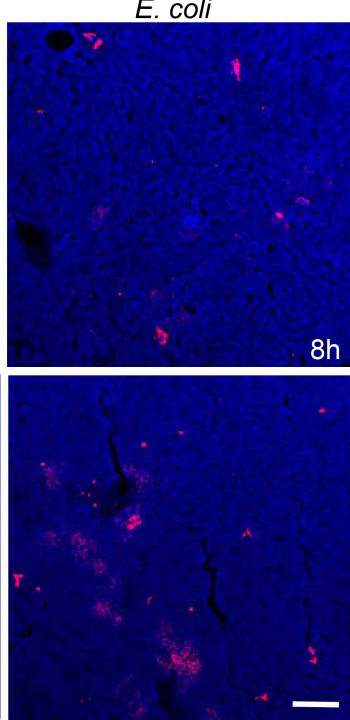

$8 \mathrm{~h}$

Figure 3. Platelet CLEC-2 deficiency increases complement activation and liver attack in early sepsis. (A-C) Mouse plasma C3a, C5a, and C5b-9 levels measured by ELISA. Data are shown as mean \pm SD. $n=9$ mice/group. (D) Representative images of C5b-9 complex staining of liver cryosections at 8 hours after injection of $E$. coli or saline. (E) Representative images of TUNEL staining of liver cryosections. Bar graphs below were quantifications of C5b-9- or TUNEL-positive cells. hpf, high-power microscopic fields. The data are mean $\pm \mathrm{SD} . n=6$ mice/group. ${ }^{*} P<0.05$, comparing the sample at 0 hours of $E$. coli injection with samples at other time points after $E$. coli infection. ${ }^{*} P<0.05$. ${ }^{* *} P<0.01$. ${ }^{* *} P<0.001$. Data were analyzed using 1 -way ANOVA followed by Tukey's test for multiple groups and were representative of 3 independent experiments. Scale bar: $10 \mu \mathrm{m}$ (D), $25 \mu \mathrm{m}$ (E).

Circulating monocytes in early sepsis express podoplanin that binds to platelet CLEC-2 and induces release of complement inhibitors from platelets. Macrophages in the lungs of septic mice express podoplanin (20), so we asked whether circulating monocytes express podoplanin to bind platelet CLEC-2. Blood was collected from mice before and after $E$. coli infection, and expression of podoplanin on monocytes was detected by flow cytometry. Ly6C and CD11b double-positive cells were defined as monocytes (Supplemental Figure 10). In healthy animals, podoplanin-positive monocytes were negligible (Figure $5 \mathrm{~A}$ ). Infection with $E$. coli increased the percentages of podoplanin-positive monocytes to around $15 \%$ and $35 \%$ at 0.5 hour and 1 hour, respectively (Figure $5 \mathrm{~A}$ ). The percentage of podoplanin-positive monocytes declined at 2 hours and 4 hours after infection (Figure 5A). Monocytes in Plt Clec2-/- mice showed a similar profile of podoplanin expression as those in WT mice (Figure 5A), indicating that podoplanin expression on monocytes in the septic mice was independent of platelet CLEC-2. 
A

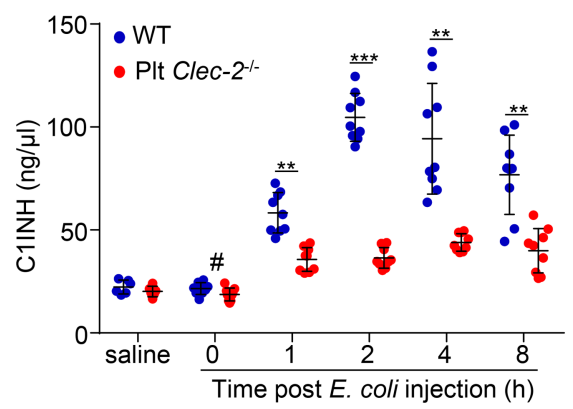

D

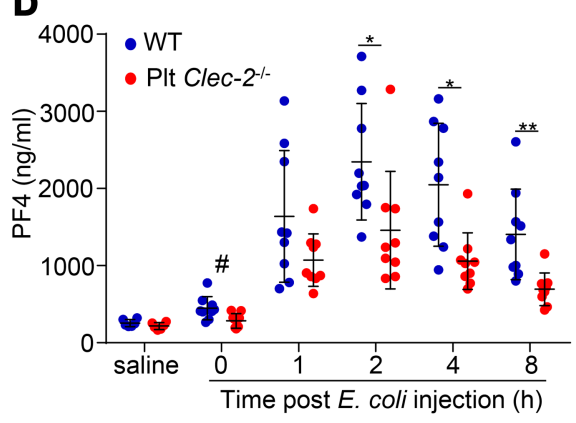

B

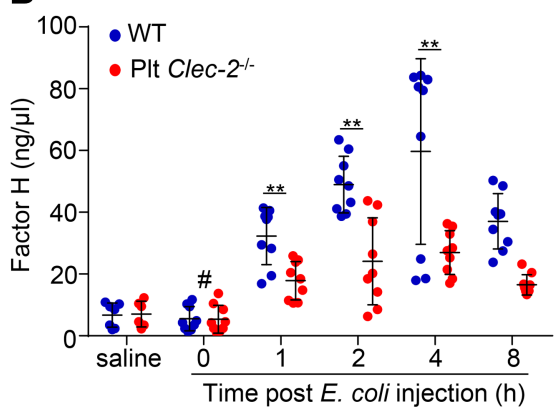

C

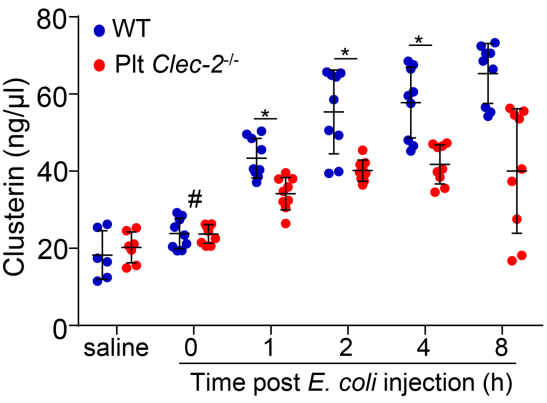

E

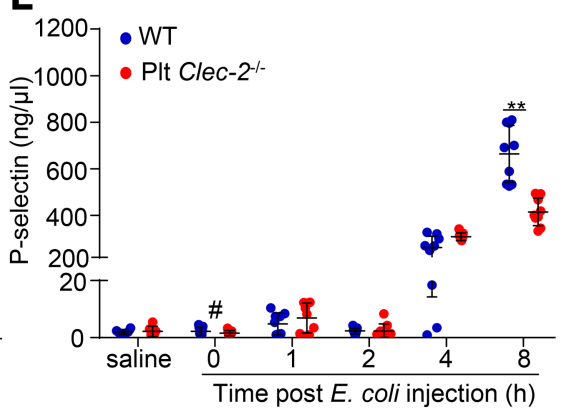

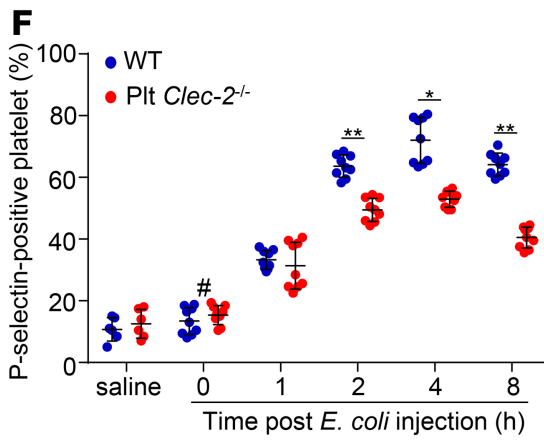

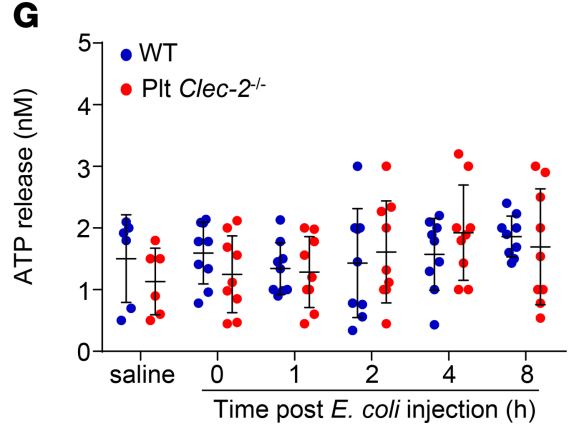

Figure 4. Platelet CLEC-2 deficiency reduces release of complement inhibitors from platelets in early sepsis. (A-E and C) Plasma levels of different complement inhibitors, PF4, P selectin, and ATP in WT and PIt Clec-2-1- mice with or without E. coli injection. (F) P selectin-positive platelets in WT and PIt Clec $-2^{-1-}$ mice with or without $E$. coli injection. The data represent mean $\pm S D, n=9$ mice/group. ${ }^{\#} P<0.05$, comparing the sample at 0 hours of $E$. coli injection with samples at other time points after $E$. coli infection. ${ }^{*} P<0.05$. ${ }^{* *} P<0.01$. ${ }^{* *} P<0.001$. Data were analyzed using 1 -way ANOVA followed by Tukey's test for multiple groups and were representative of 3 independent experiments.

Further flow cytometry analysis showed that $E$. coli infection increased platelet-bound monocytes in the blood of WT mice relative to its basal level (Figure 5B and Supplemental Figure 11), indicating that podoplanin on blood monocytes interacts with platelet CLEC-2 in early sepsis to form platelet and monocyte aggregates. At 1 hour and 8 hours after $E$. coli infection, there were more aggregates in WT mice than Plt $\mathrm{Clec}^{-1-}$ mice (Figure 5B), suggesting more platelet activation in WT mice at these time points. We then confirmed the effect of podoplanin binding on the release of complement inhibitors from platelets. Incubation of isolated WT platelets with recombinant podoplanin, but not the vehicle control, rapidly induced secretion of C1-INH in a dose-dependent manner (Supplemental Figure 12, A and B). The selected dose of podoplanin caused secretion of PF4, C1-INH, clusterin, and factor $\mathrm{H}$ from WT platelets but not $\mathrm{Clec}^{-1-}$ platelets (Figure 5, C-F). Thus, podoplanin binding induced CLEC-2-dependent release of complement inhibitors from platelet $\alpha$-granules.

Myeloid podoplanin deficiency reduces plasma levels of complement inhibitors and enhances liver dysfunction in early sepsis. To confirm the role of monocyte podoplanin in complement inhibition and liver protection in sepsis, we used LysM Pdpn ${ }^{-/-}$mice that had a normal platelet count in the peripheral blood (Supplemental Figure 13A). E. coli injection induced expression of podoplanin in circulating monocytes of WT but not LysM Pdpn ${ }^{-/-}$mice (Supplemental Figure 13, B-E), confirming the deletion of podoplanin in blood monocytes. Infection with a half-lethal dose of E. coli caused a higher mortality in LysM 
A

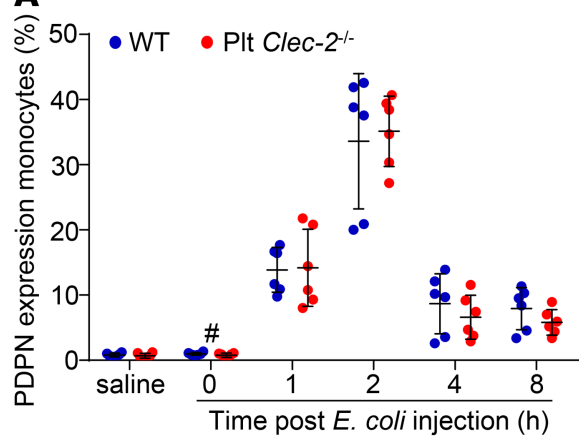

D

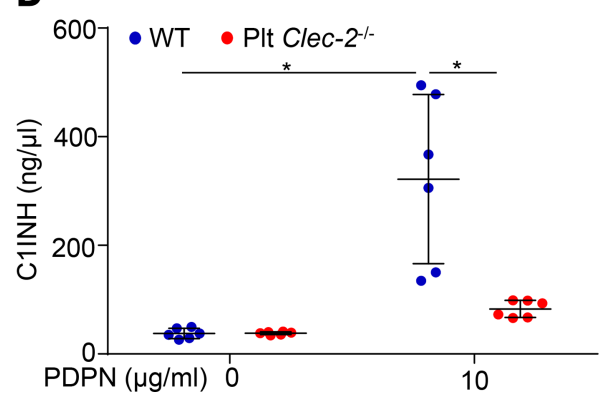

B

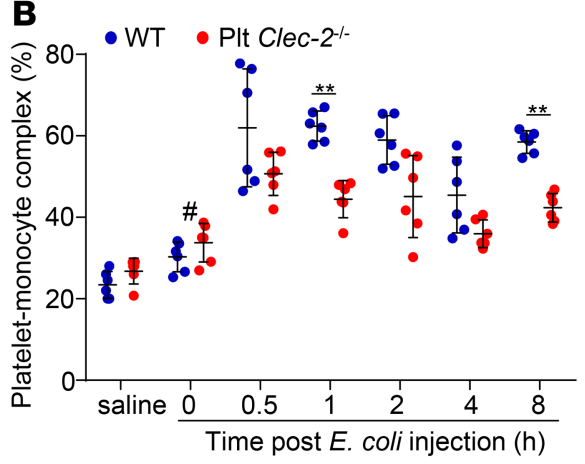

E

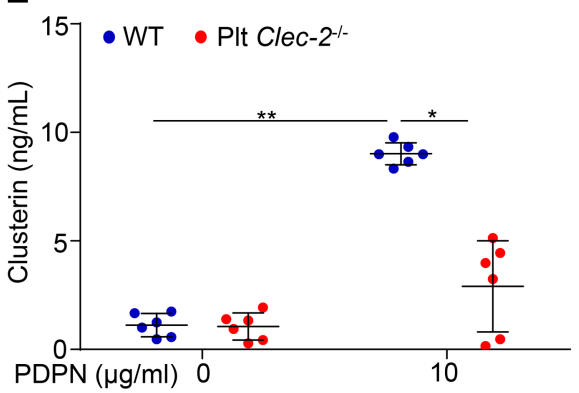

C

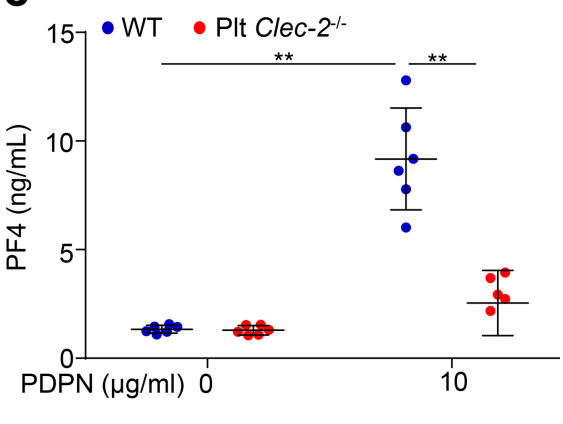

F

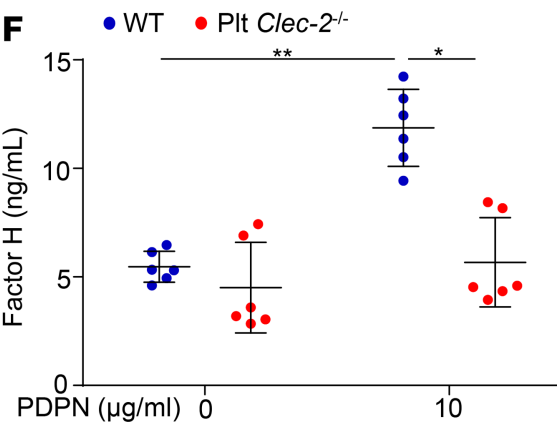

Figure 5. Podoplanin expressed on circulating monocytes binds to CLEC-2 and induces platelet release of complement inhibitors in early sepsis. (A) Podoplanin expression on monocytes and (B) monocyte-platelet aggregates from peripheral blood of mice with or without $E$. coli injection were analyzed by flow cytometry. The platelet-monocyte complex was shown as the percentage of total monocytes. (C-F) Levels of PF4, C1-INH, clusterin, and factor $\mathrm{H}$ released from WT and Clec $-2^{-1-}$ platelets incubated with saline or recombinant podoplanin for 2 hours at $37^{\circ} \mathrm{C}$. Data represent mean $\pm S D, n=6$ mice $/$ group. ${ }^{\#} P<0.05$, comparing the sample at 0 hours of $E$. coli injection with samples at other time points after $E$. coli infection. ${ }^{*} P<0.05$. ${ }^{* *} P<0.01$. Data were analyzed using 1-way ANOVA followed by Tukey's test for multiple groups and were representative of 3 independent experiments.

$P d p n^{-1-}$ mice (Figure 6A). The serum levels of LDH, bilirubin, ALT, and AST in LysM Pdpn ${ }^{-1}$ mice were higher than those in WT mice at 8 hours after E. coli infection (Figure 6, B-E), whereas the plasma levels of C1-INH, factor $\mathrm{H}$, and clusterin in LysM Pdpn ${ }^{-1-}$ mice were lower than those in WT mice at 1 hour and/or 2 hours after E. coli infection (Figure 6, F-H). Consequently, the plasma levels of C5a, C3a, and C5b-9 in LysM Pdpn ${ }^{-1-}$ mice were higher than those in WT mice after E. coli infection (Figure 7, A-C). Thus, LysM Pdpn $n^{-/-}$mice recapitulated the phenotype of Plt $\mathrm{Clec}^{-/-}$mice. These results indicate that interaction between monocyte podoplanin and platelet CLEC-2 is important in complement activation-dependent liver dysfunction in early sepsis.

Injection of complement inhibitors released from platelets protects liver function in early sepsis. Next, we asked whether injection of complement inhibitors from platelets can protect liver function in septic mice. We incubated WT and $\mathrm{Clec}^{-1-}$ platelets with a podoplanin-Fc chimera, then removed podoplanin from the supernatant using protein A/G beads (Supplemental Figure 14). Complement inhibitors, such as C1-INH, were secreted from WT platelets, but not $\mathrm{Clec}^{-{ }^{--}}$platelets (Supplemental Figure 15). We injected the supernatant of stimulated WT and $\mathrm{Clec}^{---}$platelets into Plt Clec2 ${ }^{--}$mice at 1 hour after E. coli infection, and collected blood and the liver at 8 hours after infection. As expected, injection of the supernatant from podoplanin-treated WT platelets, but not $\mathrm{Clec}^{-1-}$ platelets or saline-treated WT platelets, increased the plasma level of C1-INH (Figure 8A). Injection of the supernatant from WT platelets also reduced the serum levels of ALT, bilirubin, and LDH, and downregulated gene expression of TNF- $\alpha$ and IL-1 $\beta$ in the livers of septic mice (Figure 8, B-F). Thus, transfusion of the supernatant from podoplanin-treated WT platelets increased the plasma levels of complement inhibitors in Plt $\mathrm{Clec}-2^{-/}$mice after E. coli infection, which contributed to improved liver function in early sepsis.

\section{Discussion}

We have shown that platelets protect liver function in a mouse model of E. coli sepsis by inhibiting complement activation. Circulating monocytes expressed podoplanin, which bound to CLEC-2 and induced 
A

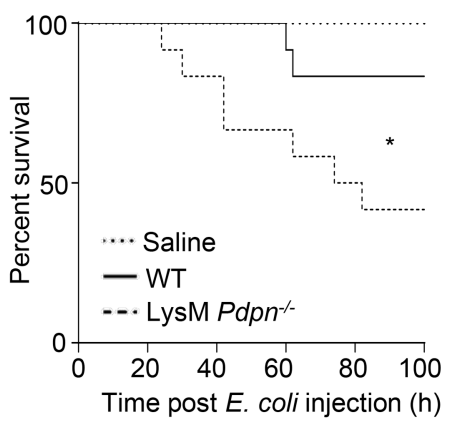

B

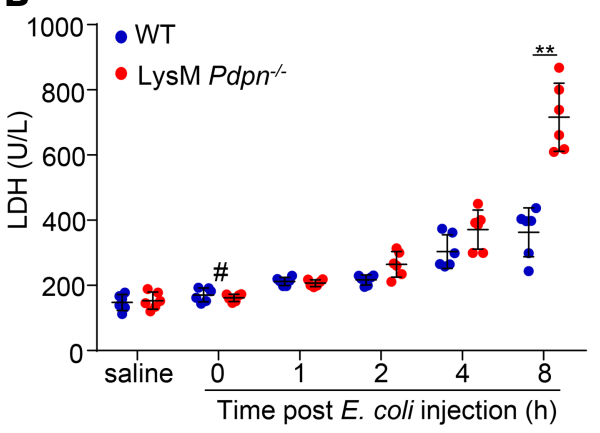

E

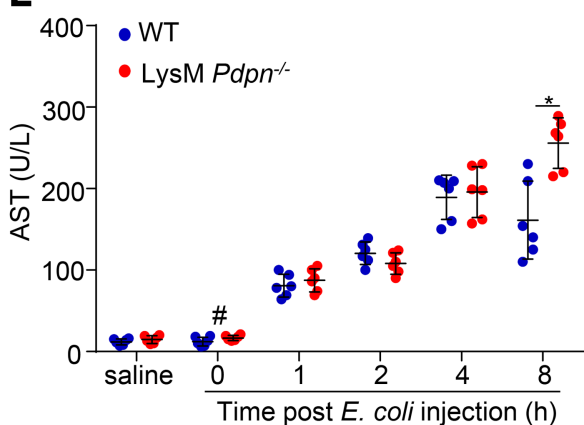

C
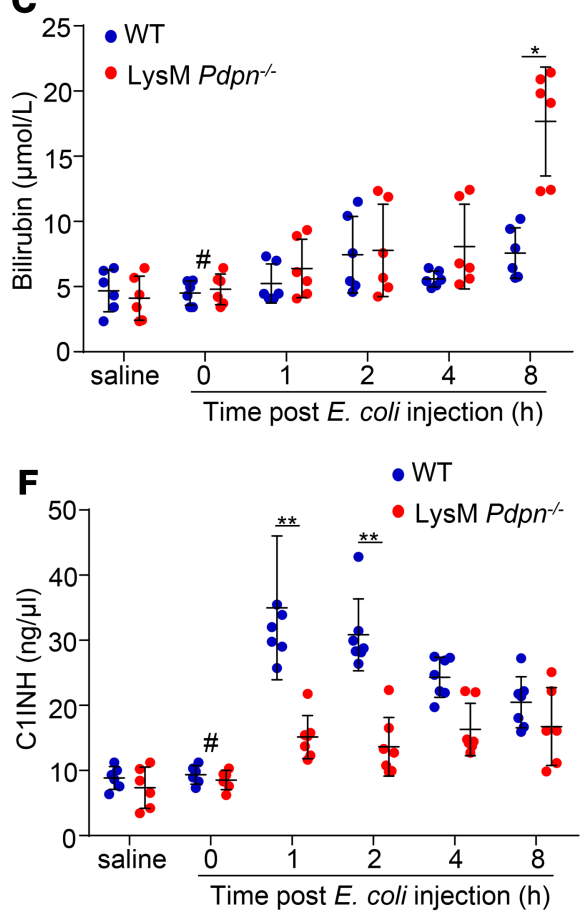

D
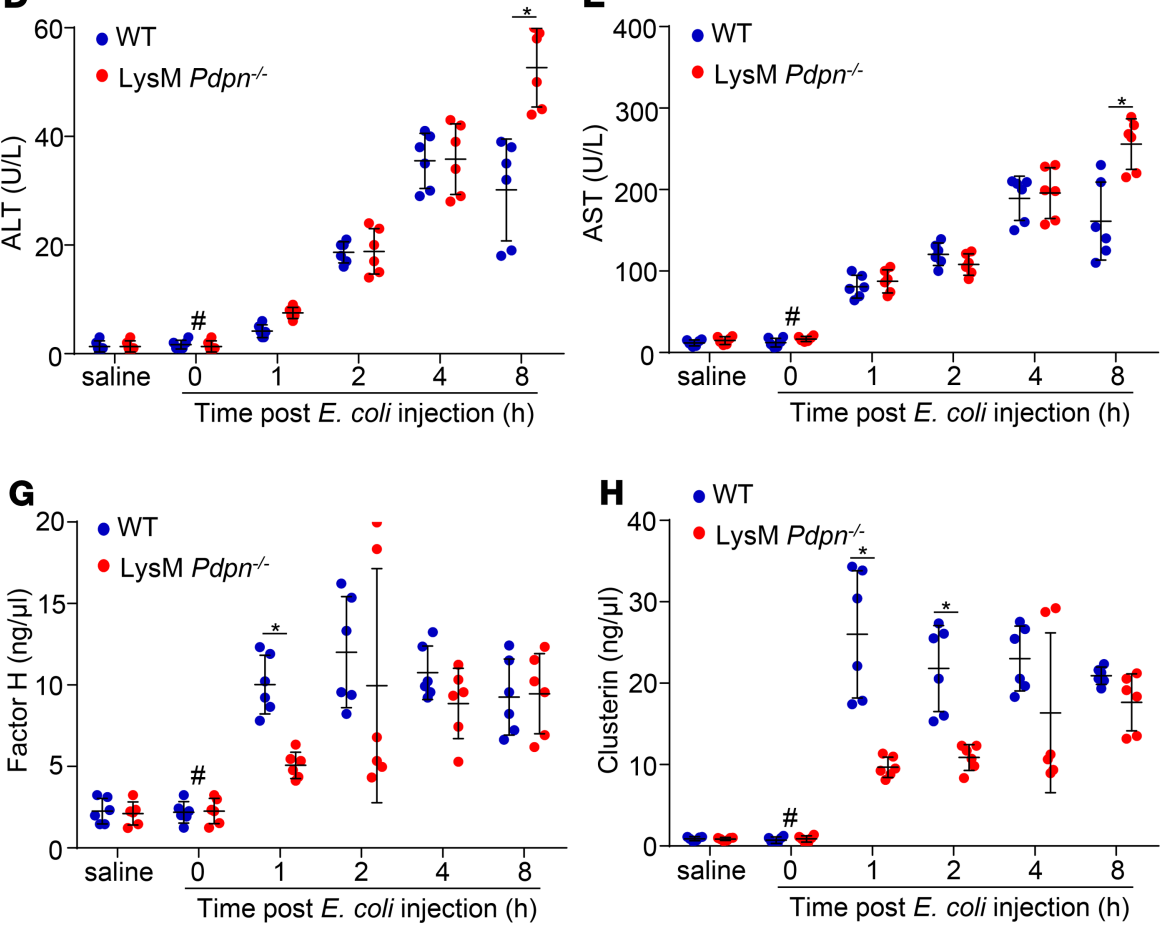

Figure 6. Myeloid podoplanin deficiency reduces plasma levels of complement inhibitors and exacerbates liver dysfunction in early sepsis. (A) The survival rate of WT and LysM Pdpn ${ }^{-1-}$ mice with or without $E$. coli infection. $n=15$ mice/group. Data were analyzed using a log-rank test and representative of 3 independent experiments. (B-H) Plasma levels of liver enzymes or platelet complement inhibitors of mice with or without $E$. coli injection. Data represent mean \pm SD; $n=6$ mice/group. ${ }^{~} P<0.05$, comparing the sample at 0 hours of $E$. coli injection with samples at other time points after $E$. coli infection. ${ }^{*} P<0.05 .{ }^{*} P<0.01$. Data were analyzed using 1-way ANOVA followed by Tukey's test for multiple groups and were representative of 3 independent experiments.

secretion of complement inhibitors from platelets in early sepsis. These inhibitors reduced complement activation in the liver and consequently ameliorated liver dysfunction.

Septic liver injury progresses from hepatocellular dysfunction to liver failure. As an early sign of sepsis, liver dysfunction occurs within a couple of hours in a mouse sepsis model and within 24 hours in patients after diagnosis (8). Inflammation, coagulation, and complement cross-interact with each other and contribute to liver injury differentially at the different stages of sepsis. Inhibition of complement activation reduces coagulation activation and ameliorates systemic inflammatory responses within 24 hours in a primate model of $E$. coli sepsis, which protects liver function as a consequence (26). Uncontrolled complement activation causes cell death and organ damage in sepsis (24). However, it is unclear how complement activation is controlled autonomously in sepsis. Our results showed that platelets were a major source of complement inhibitors in early sepsis. Released complement inhibitors from platelets protected organs in septic animals from complement attack and complement-induced inflammation. These results not only are consistent with a primate study that shows inhibition of complement $\mathrm{C} 3$ and $\mathrm{C} 5$ protects liver function (35) but also provide mechanistic insights into the pathological consequences of the activated complement in early sepsis. 
A

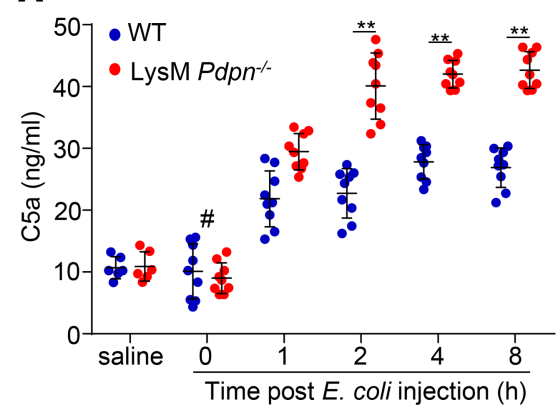

B

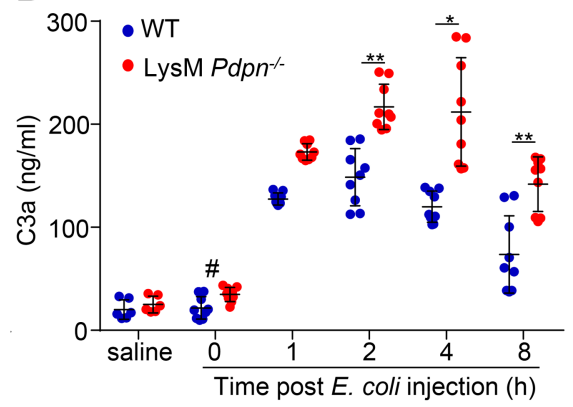

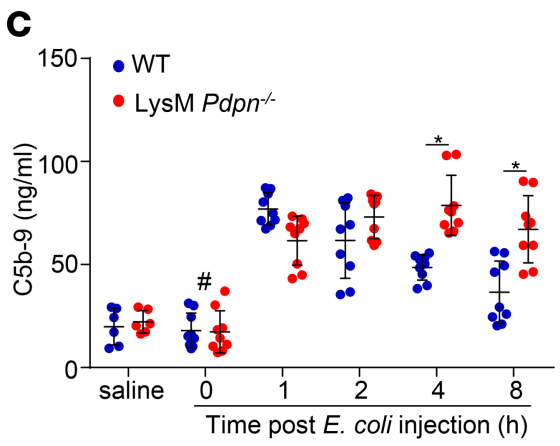

Figure 7. Deficiency of myeloid cell podoplanin increases complement activation in early sepsis. WT and LysM Pdpn ${ }^{-/-}$mice were i.p. injected with a half-lethal dose of $E$. coli $\left(2 \times 10^{7} \mathrm{CFU} /\right.$ mouse). Peripheral blood was collected from mice without $E$. coli injection or at different time points after E. coli infection. The complement activation was determined by measuring the plasma levels of C5a (A), C3a (B), and C5b-9 (C) with ELISAs ( $n=9$ mice/group). The data were presented as mean \pm SD. ${ }^{P} P<0.05$, comparing the sample at 0 hours of $E$. coli injection with samples at other time points after $E$. coli infection. ${ }^{*} P<0.05$. ${ }^{*} P<0.01$. Data were analyzed using 1-way ANOVA followed by Tukey's test for multiple groups and were representative of 3 independent experiments.

Our results showed that liver macrophages including the Kupffer cells of healthy mice and mice at the early stage of E. coli sepsis did not express podoplanin. This is consistent with previous reports that podoplanin is only expressed on lymphatic endothelial cells, mesothelial cells (36), and some progenitor cells (37) in the liver after prolonged stimulations, such as CCL4 administration and peritoneal dialysis $(37,38)$. A recent study shows that podoplanin is expressed by multiple macrophage populations in the liver during Salmonella Typhimurium infection (39). However, podoplanin expression in the Salmonella Typhimurium infection model occurs mainly around day 7 of infection, which is much later than the stage we focused on in the current study. Macrophages are highly heterogenous in terms of their functions and origins. At day 7 of infection, some podoplanin-positive macrophages may be derived from infiltrated circulating monocytes. In addition, different types of infection may account for different podoplanin expression patterns of macrophages in response to inflammation.

The role of platelets in the pathogenesis of sepsis is unclear. In a canonical model, activated platelets promote thrombosis and inflammation, facilitating organ injury in sepsis (40-42). However, platelet transfusion (15-17), but not platelet antagonists $(13,14)$, shows beneficial effects in sepsis management. It is thought that platelet transfusion protects septic animals by inhibiting inflammatory cytokine generation $(17,18)$; however, antiinflammation does not benefit the therapy of patients with sepsis (21). Thus, reduced inflammatory responses may not be the major contributor for the protective effect of platelet transfusion. Our studies demonstrated that platelets are a major resource of complement inhibitors in early sepsis. Injection of complement inhibitors into septic mice rescued liver function and reduced inflammation in the liver, indicating that supplementation of complement inhibitors was a major protective factor of platelet transfusion in sepsis. There was a modest increase of complement inhibitors in septic Plt Clec-2-- mice, suggesting podoplanin-dependent platelet release may not be the only source for complement inhibitors. Cell types such as monocytes (43), endothelial cells (44), and liver parenchymal cells (45) can synthesize C1-INH (46). Nevertheless, substantial reduction of the

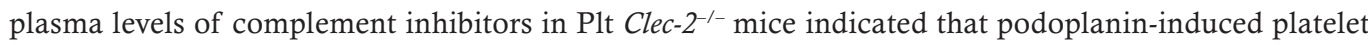
release was a primary source for the complement inhibitors in early sepsis. Our results also suggest that the inconsistent effects of platelet antagonists in sepsis treatment may be due to the blockade of platelet activation and the release of complement inhibitors. Podoplanin binding to CLEC-2 activates platelets and promotes the release of platelet granules (47-49). This finding agrees with previous reports that formation of platelet-monocyte aggregates is an early phenomenon in sepsis progression (50,51). Thus, CLEC-2-dependent platelet activation and granule release occur in the blood during early sepsis mainly through the interaction with podoplanin on monocytes.

In summary, platelets are an important source of complement inhibitors and protect liver function in early sepsis by inhibiting complement activation. We suggest a potentially novel mechanism underlying the protection of liver function in early sepsis. 
A

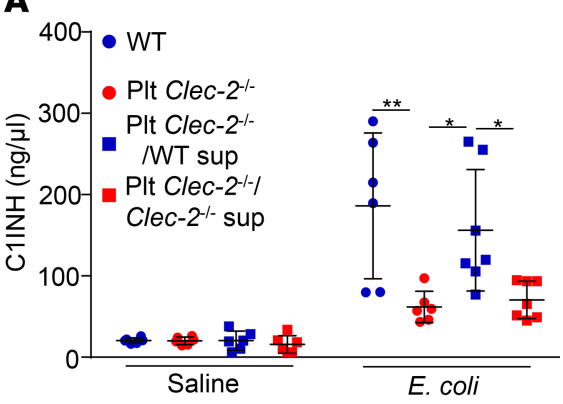

D

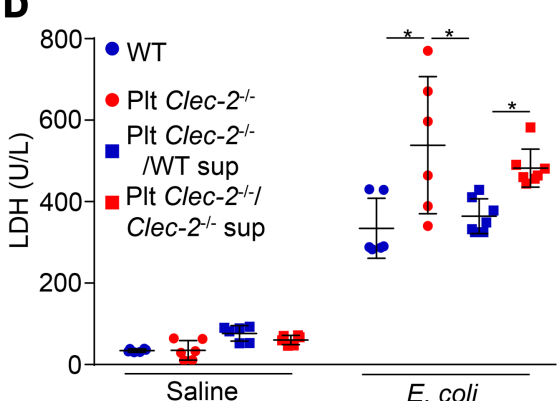

B
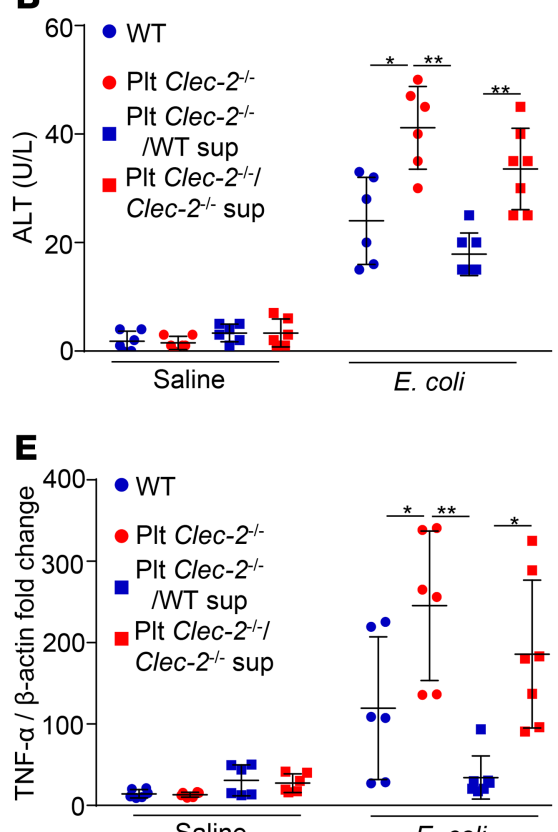

C

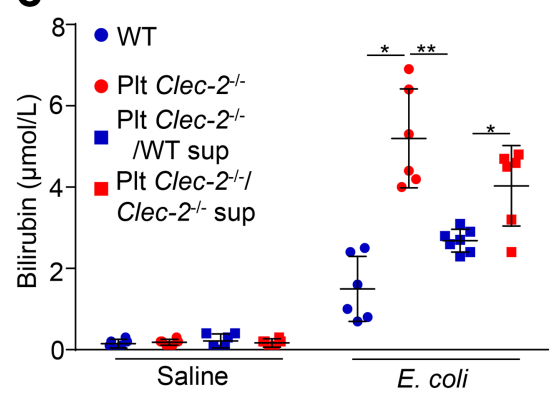

$\mathbf{F}$

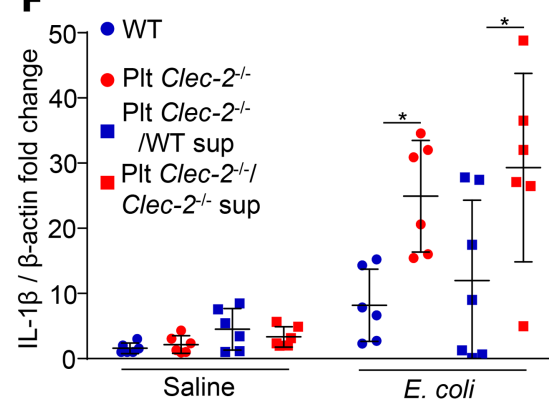

Figure 8. Injection of complement inhibitors released from platelets protects liver function in early sepsis. Plasma levels of (A) complement inhibitors, (B-D) liver function biomarkers, and (E and F) mRNA transcripts of the liver of WT or mutant mice injected with $E$. coli or saline for 8 hours. Some infected mutant mice were injected with the supernatant of podoplanin-pretreated WT or Clec-2 ${ }^{-1-}$ platelets at 1 hour after E. coli infection. Data represent mean $\pm \mathrm{SD}$. ${ }^{*} P<0.05$. ${ }^{*} P<0.01 . n=6$ mice/group. Data were analyzed using 2-way ANOVA followed by Tukey's test for multiple groups and were representative of 3 independent experiments.

\section{Methods}

Reagents. E. coli (ATCC25922) was a gift from Yi Wu (Cyrus Tang Hematology Center, Collaborative Innovation Center of Hematology, Soochow University, Suzhou, China). ELISA kits to detect mouse C5a (ELM-CCC5a-1) (52) and P selectin (ELM-Pselectin1) were purchased from Raybio. ELISA kits for C3a (MBS268280) (53) and C5b-9 (MBS776999) (54) were purchased from MyBioSource. ELISA kit for factor $\mathrm{H}$ was from Cusbio (CSB-E08933m) (55). ELISA kit for C1-INH was from Novus (NBP2-70029). ELISA kits for clusterin (ab199079) (56) and PF4 (ab100735) (57) were from Abcam. ApopTag fluorescein in situ apoptosis detection kit (S7110) was purchased from Millipore. Recombinant mouse podoplanin-Fc chimera protein (3244-PL-050) was from R\&D Systems, Bio-Techne. Chicken polyclonal anti-albumin antibody (ab106582), rat monoclonal anti-F4/80 (ab6640), rabbit polyclonal anti-C5b-9 antibody (ab55811), rabbit polyclonal anti-TNF- $\alpha$ antibody (ab6671), rabbit polyclonal anti-IL-1 $\beta$ antibody (ab2105), Syrian hamster monoclonal anti-podoplanin antibody (ab11936), Alexa Fluor 405-conjugated goat anti-chicken IgY (ab175674), Alexa Fluor 594-conjugated goat anti-rat IgG (ab150160), Alexa Fluor 647-conjugated donkey anti-rabbit IgG (ab150075), and Alexa Fluor 647-conjugated goat anti-Syrian hamster IgG (ab180117) were purchased from Abcam. PerCP anti-mouse CD11b antibody (clone: M1/70) and phycoerythrin (PE) anti-mouse Ly6C antibody (clone: HK1.4) were from BioLegend. FITC anti-mouse podoplanin antibody (clone: 8.1.1) and FITC anti-mouse CD41 antibody (clone: MWReg30) were from eBioscience, Thermo Fisher Scientific. Allophycocyanin-conjugated anti-CD11b (17-0112-81) and PE-labeled antibodies, including anti-B220 (12-045281), anti-CD49b (12-5971-63), anti-NK1.1 (12-5921-81), and anti-Ly6G (12-9668-82), were from eBioscience, Thermo Fisher Scientific. PE-labeled anti-CD90 (MA5-17749) was from Invitrogen, Thermo Fisher Scientific. Anti-PE microbeads (130-048-801) were from Miltenyi Biotec. PE anti-mouse CD41 antibody (558040) was from BD Biosciences. Polybead microspheres $3.00 \mu \mathrm{m}$ (17134-15) were from Polysciences.

Mice and sepsis models. C57BL/6J (B6, CD45.2) mice, Pf4-Cre mice, and LysM-Cre mice were purchased from The Jackson Laboratory. Clec-2-floxed mice $\left(C l e c-2^{f / f f}\right)$ and podoplanin-floxed mice $\left(P d p n^{f l / f}\right)$ were generated as previously described (27). Platelet $C L E C$-2-deficient mice (Plt $C l e c 2^{-1-}$ ) were generated by crossing

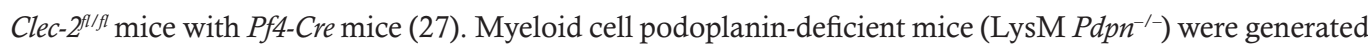
by crossing $P d p n^{f / f l}$ mice with LysM-Cre mice. 
Plt $\mathrm{Clec}^{-/-}$or LysM Pdpn ${ }^{-/-}$and WT littermates at 8 weeks old were i.p. injected with a half-lethal dose of E. coli $\left(2 \times 10^{7} \mathrm{CFU} /\right.$ mouse $)$. At different time points after injection, animals were weighed, and blood and tissues were collected. Animal survival was observed for 100 hours.

Platelet count with flow cytometry. Peripheral blood was collected and counted by flow cytometry using $\mathrm{PE}$ anti-mouse CD41 antibodies and Polybead microspheres $3.00 \mu \mathrm{m}$ (Polysciences) as internal standards.

Blood chemistry. Blood was collected by cardiac puncture without anticoagulant, and serum was isolated by centrifugation at $2000 \mathrm{~g}$. Serum bilirubin, AST, LDH, and ALT were measured by an automatic chemistry analyzer (Roche Cobas 8000).

Histopathology. Mouse liver tissues were fixed, processed, and embedded in paraffin. After removal of the paraffin, tissues were sectioned ( $5 \mu \mathrm{m} / \mathrm{section})$ and stained with H\&E (Beyotime Products). Slides were imaged with a Leica DM2000 microscope.

Quantitative PCR. Total RNA from mouse livers was extracted with the RNeasy Plus Mini Kit (QIAGEN). cDNA was prepared from $1.8 \mu \mathrm{g}$ RNA in $20 \mu \mathrm{L} \mathrm{H}_{2} \mathrm{O}$ using the High Capacity RNA-to-cDNA Kit (Life Technologies). The levels of TNF- $\alpha$ and IL-1 $\beta$ cDNA were quantitated by quantitative PCR using IQ SYBR Green Supermix (Bio-Rad) in $20-\mu \mathrm{L}$ reactions under conditions of 1 cycle at $50^{\circ} \mathrm{C}$ for 2 minutes, 1 cycle at $95^{\circ} \mathrm{C}$ for 2 minutes, and 40 cycles at $95^{\circ} \mathrm{C}$ for 15 seconds and $60^{\circ} \mathrm{C}$ for 35 seconds. The primers were CTACTCCCAGGTTCTCTTCAA and GCAGAGAGGAGGAGGTTGACTTTC for TNF- $\alpha$; TGTGTCTTTCCCGTGGACCT and CAGCTCATATGGGTCCGACA for IL-1 $\beta$ (58). Actin cDNA was used as internal control. Primers for actin were GTGCTATGTTGCTCTAGACTTCG and ATGCCACAGGATTCCATACC (58). Data from at least 3 independent sets were calculated as mean \pm SD.

Confocal analysis of the septic liver. Livers of septic mice were collected at indicated time points after infection with $E$. coli and fixed with $4 \%$ paraformaldehyde. After cryoprotection with $20 \%$ sucrose, livers and other organs were embedded with OCT compound (Tissue-Tek, Sakura Finetek). Cryosections of $15-\mu \mathrm{m}$ thickness were prepared and stained with antibodies against albumin, macrophages (F4/80), C5b-9, TNF- $\alpha$, or podoplanin. Samples were imaged with a confocal microscope (TCSSP8, Leica).

Measurement of blood complement components and complement inhibitors. Blood was collected from the retro-orbital plexus at different time points after $E$. coli infection. The plasma levels of C3a, C5a, C5b-9, C1-INH, factor $\mathrm{H}$, and clusterin were measured by ELISA kits following the manufacturer's instructions. The plasma level of PF4 was measured with ELISA kits as controls for platelet granule secretion. ATP release tracings from the same blood samples were performed with the Lumi-Aggregometer model 700 (Chrono-log).

TUNEL assay. For in situ analysis of apoptotic cell death in the septic liver, livers were immersion fixed in $10 \%$ buffered formalin and embedded in paraffin. After tissues were sectioned and deparaffinized, sections were stained using the TUNEL kit (MilliporeSigma). Total cells with FITC-labeled apoptotic nuclei were counted and analyzed under a fluorescence microscope.

Analysis of platelet-monocyte aggregates and monocyte expression of podoplanin. Anticoagulated mouse blood was collected via cardiac puncture and was diluted (1:1) with Dulbecco's PBS (DPBS) without calcium and magnesium. After lysis of red blood cells, total white blood cells were stained with fluorophore-tagged antibodies. Platelet-monocyte aggregate and podoplanin expression on monocytes were analyzed with FACSCalibur (Beckman Coulter FC500). Specifically, the white blood cell population was selected with size scatter. In the white cell population, cells that were double positive for CD11b and Ly6C were defined as circulating monocytes, and podoplanin expression in this population was measured. In monocytes, cells that were triple positive for CD11b, Ly6C, and CD41 were defined as the platelet-monocyte aggregate. The complexes show as the percentage of all monocytes of double-positive CD11b and Ly6C.

Complement inhibitor secretion from platelets in vitro. Mouse blood was collected via cardiac puncture with a 21-G needle and a syringe containing $200 \mu \mathrm{L}$ of acid citrate dextrose (ACD: $2.5 \%$ trisodium citrate, $2.0 \%$ D-glucose, $1.5 \%$ citric acid), followed by 1:1 dilution with modified Tyrode's buffer (134 mM NaCl, $0.34 \mathrm{mM} \mathrm{Na}_{2} \mathrm{HPO}_{4}$, $2.9 \mathrm{mM} \mathrm{KCl}, 12 \mathrm{mM} \mathrm{NaHCO}_{3}, 20 \mathrm{mM}$ HEPES at $\mathrm{pH}$ 7.0, $5 \mathrm{mM}$ glucose, 0.35\% BSA). Blood was centrifuged at $100 \mathrm{~g}$ for 8 minutes, and the platelet-rich plasma (PRP) was transferred to a new tube. PRP was centrifuged at $800 \mathrm{~g}$ for 10 minutes at room temperature (RT) to obtain a platelet pellet. The platelet pellet was washed twice in $1 \mathrm{~mL}$ modified Tyrode's buffer and resuspended with modified Tyrode's buffer. Washed platelets were incubated with different doses of podoplanin for different times; then platelets were removed by centrifugation at $800 \mathrm{~g}$ for 10 minutes. Complement inhibitors, PF4, and P selectin in the supernatant were measured with ELISA kits.

Rescue of liver function in septic mice with secreted complement inhibitors from platelets. WT and CLEC-2-deficient platelets were incubated with $10 \mu \mathrm{g} / \mathrm{mL}$ recombinant podoplanin-Fc chimera for 2 hours at $37^{\circ} \mathrm{C}$. 
After spinning down platelets, the supernatant was incubated with protein A/G agarose beads at RT for 1 hour to remove podoplanin. The concentration of C1-INH was measured with an ELISA kit, and the removal of podoplanin was confirmed with Western blot. Platelet CLEC-2-deficient mice at 8 weeks old were i.p. injected with a half-lethal dose of E. coli. At 1 hour after infection, the supernatant from podoplanin-pretreated WT and CLEC-2-deficient platelets was i.p. injected into septic mice. At 8 hours after infection, blood and livers were collected for the measurement of liver function and complement activation.

Detection of complement activation by zymosan-activated serum. To prepare zymosan-activated serum, $0 \mathrm{mg}$, $2.5 \mathrm{mg}, 10 \mathrm{mg}, 40 \mathrm{mg}$, and $160 \mathrm{mg}$ zymosan was diluted in $1 \mathrm{~mL}$ distilled water. The solution was centrifuged at $300 \mathrm{~g}$ for 5 minutes at RT, and the pellet was washed with DPBS. After a further centrifugation step, the supernatant was removed, and the zymosan pellet was resuspended in $20 \mathrm{~mL}$ DPBS. A total of 1 $\mathrm{mL}$ of the zymosan DPBS stock solution was centrifuged ( $350 \mathrm{~g}, 5$ minutes, RT) and the supernatant was removed. For serum activation, the zymosan pellet was resuspended in $200 \mu \mathrm{L}$ serum to a final concentration of $0 \mathrm{mg}, 0.625 \mathrm{mg}, 2.5 \mathrm{mg}, 10 \mathrm{mg}$, and $40 \mathrm{mg}$ zymosan $/ \mathrm{mL}$ serum and incubated at $37^{\circ} \mathrm{C}$ for 30 minutes to activate the complement cascade. The serum levels of C3a, C5a, and C5b-9 were measured by ELISA kits (MyBioSource).

Statistics. Data are expressed as mean \pm SD from at least 3 independent experiments unless otherwise indicated. Data were analyzed using GraphPad Prism 5. For parametric comparisons, 1-way ANOVA followed by Tukey's test for multiple groups and a 2-tailed Student's $t$ test for 2 groups were used. Survival curves were generated, and comparisons of median survival were performed using a log-rank test. A $P$ value less than 0.05 was considered statistically significant.

Study approval. Animal studies were conducted with protocols approved by the Institutional Animal Care and Use Committee of Soochow University.

\section{Author contributions}

ZX, BS, and LX conceived the project, designed experiments, analyzed the data, and wrote the manuscript. CR provided critical advice and analyzed the data. ZX, BS, CH, MM, JS, MJ, ZM, FY, JH, and XB performed experiments.

\section{Acknowledgments}

This study was supported by the Jiangsu Provincial Key Medical Center (YXZXA2016002), the Priority Academic Program Development of Jiangsu Higher Education Institutions, National Natural Science Foundation of China (81520108005 and 81800128), the Natural Science Foundation of Jiangsu Province (BK20170361), the NIH (HL128390), and the Oklahoma Medical Research Foundation.

Address correspondence to: Lijun Xia, Cardiovascular Biology Research Program, Oklahoma Medical Research Foundation, 825 NE 13th Street, Oklahoma City, Oklahoma 73104, USA. Phone: 405.271.7892; Email: lijun-xia@omrf.org.

1. Singer M, et al. The Third International Consensus Definitions for Sepsis and Septic Shock (Sepsis-3). JAMA. 2016;315(8):801810 .

2. Seymour CW, et al. Time to treatment and mortality during mandated emergency care for sepsis. N Engl J Med. 2017;376(23):2235-2244.

3. Hotchkiss RS, Nicholson DW. Apoptosis and caspases regulate death and inflammation in sepsis. Nat Rev Immunol. 2006;6(11):813-822.

4. Angus DC, van der Poll T. Severe sepsis and septic shock. NEngl J Med. 2013;369(9):840-851.

5. Gustot T, Durand F, Lebrec D, Vincent JL, Moreau R. Severe sepsis in cirrhosis. Hepatology. 2009;50(6):2022-2033.

6. Okamoto K, Tamura T, Sawatsubashi Y. Sepsis and disseminated intravascular coagulation. J Intensive Care. $2016 ; 4: 23$.

7. Canabal JM, Kramer DJ. Management of sepsis in patients with liver failure. Curr Opin Crit Care. 2008;14(2):189-197.

8. Recknagel P, et al. Liver dysfunction and phosphatidylinositol-3-kinase signalling in early sepsis: experimental studies in rodent models of peritonitis. PLoS Med. 2012;9(11):e1001338.

9. Kramer L, Jordan B, Druml W, Bauer P, Metnitz PG, Austrian Epidemiologic Study on Intensive Care, ASDI Study Group Incidence and prognosis of early hepatic dysfunction in critically ill patients--a prospective multicenter study. Crit Care Med. 2007;35(4):1099-1104.

10. Deppermann C, Kubes P. Platelets and infection. Semin Immunol. 2016;28(6):536-545.

11. Kurokawa T, Zheng YW, Ohkohchi N. Novel functions of platelets in the liver. J Gastroenterol Hepatol. 2016;31(4):745-751

12. Surewaard BGJ, et al. $\alpha$-Toxin induces platelet aggregation and liver injury during Staphylococcus aureus sepsis. Cell Host Microbe. 2018;24(2):271-284.e3. 
13. Thomas MR, et al. Platelet P2Y12 inhibitors reduce systemic inflammation and its prothrombotic effects in an experimental human model. Arterioscler Thromb Vasc Biol. 2015;35(12):2562-2570.

14. Valerio-Rojas JC, Jaffer IJ, Kor DJ, Gajic O, Cartin-Ceba R. Outcomes of severe sepsis and septic shock patients on chronic antiplatelet treatment: a historical cohort study. Crit Care Res Pract. 2013;2013:782573.

15. Lieberman L, Bercovitz RS, Sholapur NS, Heddle NM, Stanworth SJ, Arnold DM. Platelet transfusions for critically ill patients with thrombocytopenia. Blood. 2014;123(8):1146-1151

16. Pène F, Benoit DD. Thrombocytopenia in the critically ill: considering pathophysiology rather than looking for a magic threshold. Intensive Care Med. 2013;39(9):1656-1659.

17. Xiang B, et al. Platelets protect from septic shock by inhibiting macrophage-dependent inflammation via the cyclooxygenase 1 signalling pathway. Nat Commun. 2013;4:2657.

18. Rayes J, et al. The podoplanin-CLEC-2 axis inhibits inflammation in sepsis. Nat Commun. 2017;8(1):2239

19. Suzuki-Inoue K, Tsukiji N, Shirai T, Osada M, Inoue O, Ozaki Y. Platelet CLEC-2: roles beyond hemostasis. Semin Thromb Hemost. 2018;44(2):126-134.

20. Lax S, et al. Platelet CLEC-2 protects against lung injury via effects of its ligand podoplanin on inflammatory alveolar macrophages in the mouse. Am J Physiol Lung Cell Mol Physiol. 2017;313(6):L1016-L1029.

21. Remick DG. Cytokine therapeutics for the treatment of sepsis: why has nothing worked? Curr Pharm Des. 2003;9(1):75-82

22. Rittirsch D, Flierl MA, Ward PA. Harmful molecular mechanisms in sepsis. Nat Rev Immunol. 2008;8(10):776-787.

23. Thorgersen EB, et al. Inhibition of complement and CD14 attenuates the Escherichia coli-induced inflammatory response in porcine whole blood. Infect Immun. 2009;77(2):725-732.

24. Gerard C. Complement C5a in the sepsis syndrome--too much of a good thing? N Engl J Med. 2003;348(2):167-169.

25. Fattahi F, et al. Complement-induced activation of MAPKs and Akt during sepsis: role in cardiac dysfunction. FASEB J. 2017;31(9):4129-4139.

26. Keshari RS, et al. Inhibition of complement C5 protects against organ failure and reduces mortality in a baboon model of Escherichia coli sepsis. Proc Natl Acad Sci U S A. 2017;114(31):E6390-E6399.

27. Herzog BH, et al. Podoplanin maintains high endothelial venule integrity by interacting with platelet CLEC-2. Nature. 2013;502(7469):105-109.

28. Aboutaleb N, Jamali H, Abolhasani M, Pazoki Toroudi H. Lavender oil (Lavandula angustifolia) attenuates renal ischemia/reperfusion injury in rats through suppression of inflammation, oxidative stress and apoptosis. Biomed Pharmacother. 2019;110:9-19.

29. Ying WZ, Li X, Rangarajan S, Feng W, Curtis LM, Sanders PW. Immunoglobulin light chains generate proinflammatory and profibrotic kidney injury. J Clin Invest. 2019;129(7):2792-2806.

30. Offenbacher S, et al. GWAS for interleukin-1 $\beta$ levels in gingival crevicular fluid identifies IL37 variants in periodontal inflammation. Nat Commun. 2018;9(1):3686.

31. Kerrigan AM, et al. Podoplanin-expressing inflammatory macrophages activate murine platelets via CLEC-2. J Thromb Haemost. 2012;10(3):484-486.

32. Schmaier AH, Amenta S, Xiong T, Heda GD, Gewirtz AM. Expression of platelet C1 inhibitor. Blood. 1993;82(2):465-474.

33. Tschopp J, et al. Human megakaryocytes express clusterin and package it without apolipoprotein A-1 into alpha-granules. Blood. 1993;82(1):118-125.

34. Devine DV, Rosse WF. Regulation of the activity of platelet-bound C3 convertase of the alternative pathway of complement by platelet factor H. Proc Natl Acad Sci U S A. 1987;84(16):5873-5877.

35. Silasi-Mansat R, et al. Complement inhibition decreases the procoagulant response and confers organ protection in a baboon model of Escherichia coli sepsis. Blood. 2010;116(6):1002-1010.

36. Ogawa T, Li Y, Lua I, Hartner A, Asahina K. Isolation of a unique hepatic stellate cell population expressing integrin $\alpha 8$ from embryonic mouse livers. Dev Dyn. 2018;247(6):867-881.

37. Eckert C, et al. Podoplanin discriminates distinct stromal cell populations and a novel progenitor subset in the liver. Am J Physiol Gastrointest Liver Physiol. 2016;310(1):G1-12.

38. Kanda E, Takai K, Maeda Y, Shiigai T. [Flow cytometric analysis of mesothelial cells in peritoneal dialysis effluent]. Nihon Jinzo Gakkai Shi. 2005;47(2):100-106.

39. Hitchcock JR, et al. Inflammation drives thrombosis after Salmonella infection via CLEC-2 on platelets. J Clin Invest. 2015;125(12):4429-4446.

40. Naime ACA, Ganaes JOF, Lopes-Pires ME. Sepsis: the involvement of platelets and the current treatments. Curr Mol Pharmacol. 2018;11(4):261-269.

41. Dewitte A, et al. Blood platelets and sepsis pathophysiology: a new therapeutic prospect in critically [corrected] ill patients? Ann Intensive Care. 2017;7(1):115.

42. Vardon Bounes F, et al. The importance of blood platelet lipid signaling in thrombosis and in sepsis. Adv Biol Regul. 2018;67:66-73

43. Johnson AM, Alper CA, Rosen FS, Craig JM. C1 inhibitor: evidence for decreased hepatic synthesis in hereditary angioneurotic edema. Science. 1971;173(3996):553-554

44. Randazzo BP, Dattwyler RJ, Kaplan AP, Ghebrehiwet B. Synthesis of C1 inhibitor (C1-INA) by a human monocyte-like cell line, U937. J Immunol. 1985;135(2):1313-1319.

45. Lappin DF, Guc D, Hill A, McShane T, Whaley K. Effect of interferon-gamma on complement gene expression in different cell types. Biochem J. 1992;281(pt 2):437-442.

46. Aw X, Li L, Wc L, Zh Y. The effect of plant growth-promoting rhizobacteria (PGPR) on arsenic accumulation and the growth of rice plants (Oryza sativa L.). Chemosphere. 2020;242:125136.

47. Ozaki Y, Suzuki-Inoue K, Inoue O. Platelet receptors activated via mulitmerization: glycoprotein VI, GPIb-IX-V, and CLEC-2. J Thromb Haemost. 2013;11(suppl 1):330-339.

48. Badolia R, Inamdar V, Manne BK, Dangelmaier C, Eble JA, Kunapuli SP. G ${ }_{\mathrm{q}}$ pathway regulates proximal C-type lectin-like receptor-2 (CLEC-2) signaling in platelets. J Biol Chem. 2017;292(35):14516-14531. 
49. Séverin S, et al. Syk-dependent phosphorylation of CLEC-2: a novel mechanism of hem-immunoreceptor tyrosine-based activation motif signaling. J Biol Chem. 2011;286(6):4107-4116

50. Gros A, Ollivier V, Ho-Tin-Noé B. Platelets in inflammation: regulation of leukocyte activities and vascular repair. Front Immunol. 2014;5:678.

51. Kral JB, Schrottmaier WC, Salzmann M, Assinger A. Platelet interaction with innate immune cells. Transfus Med Hemother. 2016;43(2):78-88.

52. Kang SA, et al. Safety evaluation of intravenously administered mono-thioated aptamer against E-selectin in mice. Toxicol Appl Pharmacol. 2015;287(1):86-92.

53. Cipolla E, et al. IL-17A deficiency mitigates bleomycin-induced complement activation during lung fibrosis. FASEB J. 2017;31(12):5543-5556.

54. Li WW, et al. Autoimmunity contributes to nociceptive sensitization in a mouse model of complex regional pain syndrome. Pain. 2014;155(11):2377-2389.

55. Xu J, et al. Function of complement factor $\mathrm{H}$ and imaging of small molecules by MALDI-MSI in a methamphetamine behavioral sensitization model. Behav Brain Res. 2019;364:233-244.

56. de Retana SF, et al. Peripheral administration of human recombinant ApoJ/clusterin modulates brain beta-amyloid levels in APP23 mice. Alzheimers Res Ther. 2019;11(1):42.

57. Petzold T, et al. $\beta 1$ integrin-mediated signals are required for platelet granule secretion and hemostasis in mouse. Blood. 2013;122(15):2723-2731.

58. Yang A, Xie Z, Wang B, Colman RW, Dai J, Wu Y. An essential role of high-molecular-weight kininogen in endotoxemia. J Exp Med. 2017;214(9):2649-2670. 